\title{
IL RAPPORTO TRA DIRITTO INTERNAZIONALE PUBBLICO E DIRITTO PETROLIFERO: STUDIO DEL CASO TRA TIMOR-LESTE E L'AUSTRALIA
}

\section{ARTICOLO ORIGINALE}

PEREIRA, Jeremias ${ }^{1}$

PITHAN, Lívia Haygert ${ }^{2}$

PEREIRA, Jeremias. PITHAN, Lívia Haygert. II rapporto tra diritto internazionale pubblico e diritto petrolifero: studio del caso tra Timor-Leste e l'Australia. Rivista scientifica multidisciplinare di nucleo di conoscenza. anno 04, Ed. 12, Vol. 02, pp. 3151. dicembre 2019. ISSN: 2448-0959, Collegamento di accesso: https://www.nucleodoconhecimento.com.br/legge/diritto-internazionalepubblico

\section{RIEPILOGO}

Questo articolo ha lo scopo di analizzare la Legge del Mare e il Petrolio per comprendere le ragioni che hanno generato, per più di un decennio, il conflitto tra Timor-Leste e l'Australia per quanto riguarda la definizione di confini marittimi definitivi. Timor-Leste è già stato sfruttato e invaso da diverse nazioni. A causa dell'abbondanza di petrolio e gas naturale, ha sofferto per ottenere l'indipendenza nel 2002, così come ha lottato per godere del suo territorio marittimo fino al 2018, da questo nuovo contesto del trattato di frontiera marittima firmato tra Timor-Leste e la Australia. La linea di equidistanza mediana è stato il parametro utilizzato per definire i confini definitivi di Timoresi, concedendo il diritto di godere del loro territorio marittimo.

1 Scuola di Diritto Accademico, Scuola di Giurisprudenza, Pontificia Università Cattolica di Rio Grande do Sul - PUC-RS.

${ }^{2}$ Consulente di orientamento. Dottorato di ricerca in Diritto Privato. Laurea magistrale in giurisprudenza. Laurea in Giurisprudenza. 
Il patto definitivo sulle frontiere ha contribuito notevolmente allo sviluppo di Timor Est, grazie allo sfruttamento delle ricchezze marine, oltre a riconoscere la necessità del paese di compagnie petrolifere che contribuiscano all'evoluzione di Timor-Leste in modo specifico e puntuale. Ciò deve ora essere ratificato dai parlamenti nazionali di entrambi i paesi. II processo di ratifica dovrebbe essere completato nel 2019. TimorLeste sta attualmente cercando di negoziare i suoi confini marittimi con l'Indonesia, ma questi sono stati sospesi durante il processo di conciliazione obbligatorio con l'Australia. Ora che questo processo è finito, i due paesi possono riprendere le discussioni.

Parole chiave: diritto petrolifero, diritto internazionale, diritto marittimo.

\section{INTRODUZIONE}

Per più di un decennio, l'Australia e Timor-Leste sono in disaccordo con i confini marittimi di ciascuno di questi Stati. La controversia si svolge a causa del diritto all'esplorazione del petrolio e di altre ricchezze marine e degli ostacoli allo sviluppo economico e politico di Timor-Leste, dopo un trattato firmato con l'Australia. Così, questo articolo si sviluppa nel settore del diritto internazionale pubblico, in particolare nel settore del diritto petrolifero.

Ci si chiede se il trattato tra l'Australia e Timor Leste, sulla definizione di frontiere marittime, sia stato equilibrato, preservando pienamente il diritto di STORdimento. Quali sono gli ostacoli per impedire che questa nazione venga sfruttata in modo sproporzionato da altre nazioni e possa svilupparsi a partire dal 2019?

La rilevanza di questa ricerca è giustificata dal fatto che la necessità di rispettare $\mathrm{i}$ confini marittimi di Timor-leste da parte dell'Australia è giustificata. Nel XXI secolo, nonostante l'esistenza del diritto internazionale e del diritto petrolifero, l'Australia non aveva alcun interesse a realizzare un trattato in cui i diritti di Timor-leste erano riconosciuti per ricevere la percentuale appropriata per l'esplorazione petrolifera di territorio marittimo. La fragilità economica storica del paese con uno spazio terrestre molto più basso del marinaio lo ha reso l'obiettivo dello sfruttamento di paesi come 
I'Indonesia e l'Australia. Sebbene nel 2018 vi sia stato un trattato permanente tra Timor-Leste e l'Australia, è necessario esplorare diversi documenti giuridici relativi alla legge marittima e petrolifera per costruire basi giuridiche internazionali, con l'obiettivo di moltiplicare gli strumenti di difesa contro altre nazioni che intendono sfruttare indebitamente lo spazio territoriale di Timor Est, a causa dell'attuale fragilità economica. ${ }^{[3]}$

Timor-Leste, fino al 1975, apparteneva al Portogallo, i suoi coloni. Dal 1976, I'Indonesia lo ha invaso e ha iniziato a sfruttarlo, e solo con l'aiuto delle Nazioni Unite (ONU) questo paese ha eletto il suo primo presidente della repubblica nel 2001, diventando uno stato indipendente e membro dell'ONU nel 2002. Nonostante l'indipendenza, Timor-Leste soffrì della tirannia australiana non riuscendo a ricevere risorse finanziarie per lo sfruttamento del suo territorio marittimo. Portogallo, Indonesia e Australia non hanno mai investito adeguatamente a Timor-Leste, anche se hanno raccolto milioni dall'estrazione di petrolio in quella regione, così come altre ricchezze. A causa di decenni di bassi investimenti finanziari, la nazione di Timorese ha bisogno di svilupparsi molto politicamente e legalmente per poter si verifichi nello sviluppo economico. ${ }^{[4]}$

L'obiettivo generale di questo documento scientifico è analizzare il diritto internazionale e petrolifero pubblico per comprendere le ragioni che hanno generato più di dieci anni fa il conflitto tra Timor-Leste e l'Australia ai confini marittimi. Per comprendere il problema che circonda il territorio marittimo di Timor Leste, sono stati delineati diversi obiettivi specifici, come

a) presentare una visione d'insieme, politica, storica, giuridica ed economica di Timor Leste e delle sue relazioni con l'Australia;

b) esaminare il diritto internazionale in materia di Mare;

c) sfruttare il diritto al petrolio;

d) analizzare il trattato sui confini tra Timor Este e l'Australia.

Questo articolo è diviso in quattro parti: le possibilità di evoluzione politico-sociale, storica, giuridica ed economica di Timor-Leste saranno analizzate nel corso della 
ricerca per raggiungere, analizzare e verificare se il trattato tra L'Australia e TimorLeste contribuiranno infatti al progresso della società di Timor. Da queste informazioni, sarà necessario comprendere le ragioni che hanno portato il popolo di Timor Est ad avere un basso livello di sviluppo in diversi settori.

Il primo capitolo tratta degli aspetti storici e geografici, oltre allo sfruttamento di TimorLeste da parte di altre nazioni, in un contesto storico, in modo da comprendere le ragioni dell'attuale contesto giuridico, economico, politico e sociale di questo paese.

Nel secondo capitolo, vengono esplorati aspetti ai confini del Mare di Timor-Leste e all'importanza delle relazioni internazionali per quel paese. Questa analisi ha la funzione di osservare i fattori che favoriscono il popolo di Timorese di essere nuovamente vittima degli esploratori, a causa della loro fragilità economica oggi. Per comprendere i meccanismi di difesa di uno Stato in relazione agli attacchi marittimi, è necessario affrontare il diritto internazionale del mare. Questa parte del diritto internazionale si occupa di standard e principi concordati a livello internazionale relativi alla proprietà, all'uso, allo sfruttamento e alla protezione del mare e delle sue risorse in tutto il mondo. ${ }^{[5]}$

Nel Terzo capitolo, il concetto e l'importanza del petrolio e la sua rilevanza per lo sviluppo di nazioni che dispongono di un enorme spazio territoriale marittimo e di un piccolo spazio terrestre, come Timor-Leste, saranno esplorati.

L'olio è stato scoperto nel XIX secolo e, fin dalla sua nascita, ci sono state importanti trasformazioni nell'umanità, $\grave{E}$ una miscela omogenea di composti organici, principalmente idrocarburi, insolubili in acqua. Questa fonte di alimentazione è anche conosciuta come grezza. Dal 1859, è stata considerata una preziosità e, nel XXI secolo, è ferocemente ambita per il ruolo rilevante che svolge nel mondo moderno. La dilagante ricerca del potere e dell'Indonesia ha portato a diversi conflitti con Timor Leste a causa dell'ambizione di alcuni paesi di sfruttare il territorio marittimo che non gli apparteneva. È estremamente importante comprendere come si verifica l'estrazione del petrolio, così come le risorse come il gas naturale, per capire le ragioni che hanno portato allo sfruttamento indebito per decenni di petrolio di Timorese. [6] 
In seguito, gli ostacoli allo sviluppo di Timor-Leste presenti nel quarto capitolo. II rapporto con la comunità internazionale è estremamente rilevante per lo sviluppo di questo paese. I progressi nella sfera giuridica e politica sono essenziali per l'evoluzione economica. Le imprese responsabili dello sfruttamento delle risorse naturali del territorio marittimo devono negoziare con professionisti timoresi preparati e formati, in modo che gli accordi offrano molti vantaggi a Timor Est. ${ }^{[7]}$

II presente studio non intende esaurire tutte le questioni, ma piuttosto stimolare le discussioni sul conflitto tra Timor e l'Australia. La tecnica di ricerca utilizzata in questo lavoro consisterà nella ricerca bibliografica, attraverso l'analisi di dottrine, articoli scientifici, biblioteche virtuali, così come la ricerca in testi legali da siti web riconosciuti come veicoli informativi accreditato dal governo di Timor-Leste. II metodo adottato sarà il processo induttivo con cui lo studente, attraverso lo studio di diverse posizioni di indottrinatori, partirà da diverse particolari conoscenze di alcuni autori, per giungere a diverse conclusioni generali. Un'esplorazione bibliografica sarà effettuata in relazione al soggetto, in particolare, con ogni sottocapitolo della sintesi per raggiungere una conclusione generale del problema. ${ }^{\left[{ }^{[3]}\right.}$

\section{TIMOR-LESTE, GEOGRAPHY, STORIA, ECONOMIA E POLITICA}

La Repubblica democratica di Timor-Leste è un paese costiero. I suoi principali prodotti economici sono le noi, il mais, il caffè, il fumo, la gomma, il legno di sandalo bianco, il bestiame, i maiali, il bufalo, tra gli altri. Questo paese si trova sull'isola di Timor e ha confini con Indonesia e Australia. II suo territorio corrisponde a sezioni di questa isola, perché nel 1975, il tempo di indipendenza del Portogallo, l'altra metà dell'arcipelago non apparteneva più al dominio portoghese. A causa di questa situazione, attualmente c'è una parte dell'isola che è il territorio dell'Indonesia. Si trova nel sud-est asiatico, sul bordo meridionale dell'arcipelago indonesiano, a nord-ovest dell'Australia, vicino all'Oceania. A sud, si trova tra le 250 e le 400 miglia nautiche attraverso il Mare di Timor con la terraferma australiana.

II territorio marittimo di questo paese, che ha un'abbondanza di petrolio e gas naturale, ha sempre attirato l'avidità dei vari paesi. I trattati e le invasioni che hanno danneggiato 
il trono di stanchezza in vari periodi storici derivano dal desiderio di invadere e assumere l'incarico per diventare un'autorità legittima per compiere lo sfruttamento del Mare di Timor-Leste. Per ottenere un vantaggio, I'Australia è stato l'unico paese al mondo che ha ufficialmente riconosciuto l'annessione illegale di Timor-Leste da parte dell'Indonesia negli anni '70, anche se c'è una risoluzione del Consiglio di sicurezza dell'Assemblea delle Nazioni Unite Nazioni Unite che hanno condannato questa invasione. ${ }^{[9]}$

Figura 1 - Didascalia immagine Confini marittimi di Timor-Leste

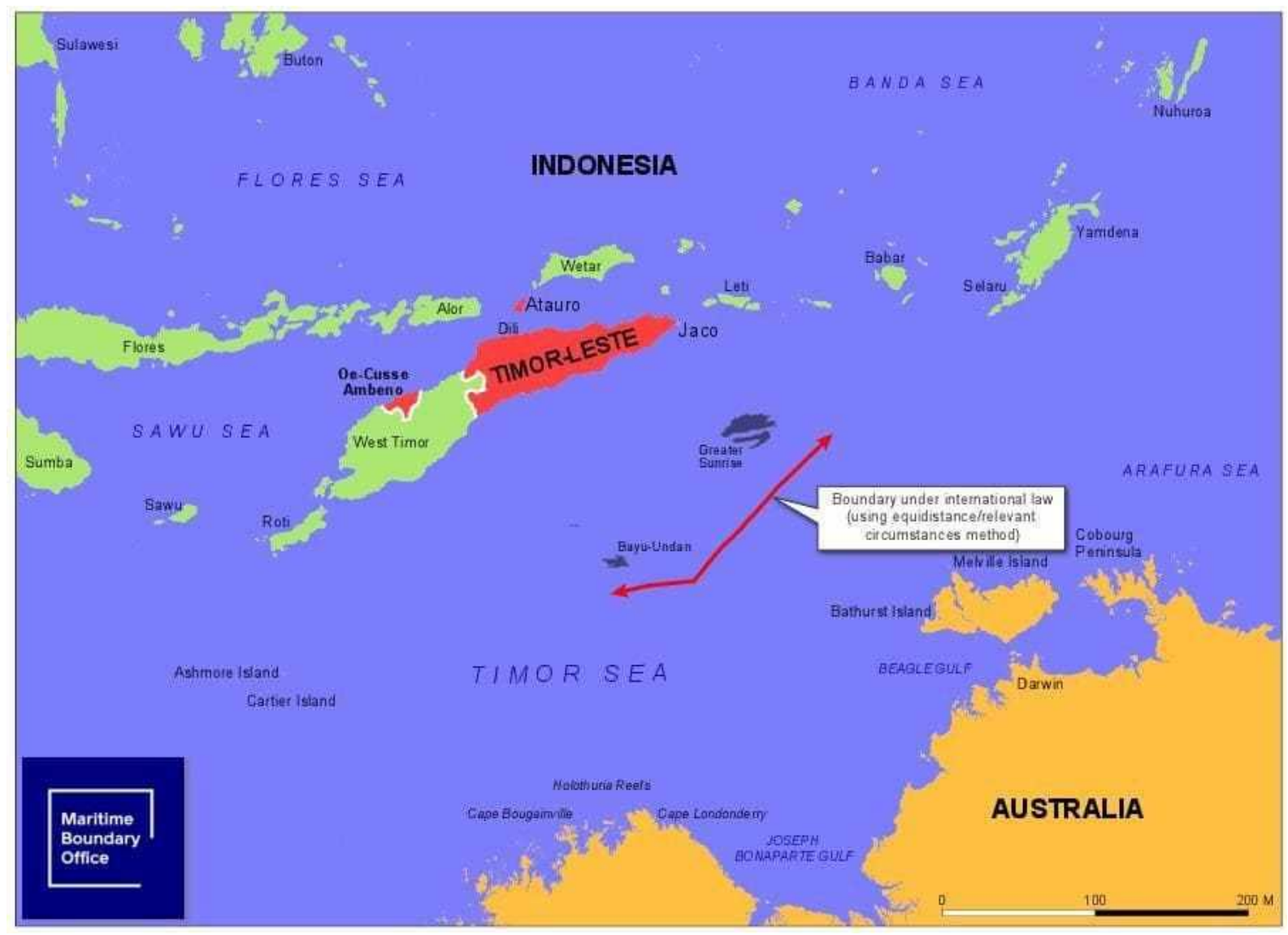

Fonte: Maritime Boundary Office (2018)[10]

Timor-Leste è già stato esplorato da Portogallo, Giappone, Indonesia e Australia in periodi diversi. II contesto storico di questa repubblica è un fattore che rivela le cause dell'attuale situazione sociale, politica ed economica di questa nazione. Questo paese, che ha un piccolo territorio terrestre e circa un milione di abitanti, ha bisogno di 
sviluppare molto in diverse aree perché è un paese giovane. II Portogallo è stato il dominatore di Timor-Leste dal 1515 al 1975 e ha investito poco nella sua crescita. Dal 1942 al 1945, nella seconda guerra mondiale, fu occupata dal Giappone, ma ben presto i portoghesi riconquistarono l'autorità di questa colonia. Nell'aprile 1975, un movimento politico delle forze armate, popolarmente noto come $3 \mathrm{D}$, emerse in Portogallo, con lo scopo di decolonizzare, democratizzare e sviluppare. Durante questo periodo, a Timor Est nacque un movimento rivoluzionario noto come FRETILIN, Fronte Rivoluzionario dell'Indipendente Timor-Leste. II 28 novembre 1975 si verificò la sua indipendenza, guidata dal Movimento di Timorese. L'indipendenza è stata osservata solo per dieci giorni. ${ }^{[11]}$

L'Indonesia, nel luglio 1975, invase Timor-Leste, rimanendo approssimativamente per ventisei anni in quel territorio, in un momento in cui si verificarono molti decessi per malattie e carenze alimentari. La comunità internazionale è stata commossa dal genocidio che vi si è verificato. Nel 1999, l'ONU, insieme al Portogallo, ha mediato un accordo con I'Indonesia, che si è occupato della possibilità di indire un referendum. Nell'agosto 1999, Timorese optò per l'indipendenza con la maggioranza dei voti, poiché il $78 \%$ della popolazione non accettò più il massacro subito. Ma i dominatori non accettavano la liberazione. II personale militare indonesiano torturava e massacrava il popolo. Molti timoresi fuggirono nella parte occidentale dell'isola, mentre tutto ciò che fu costruito lungo l'invasione fu distrutto in quel paese. Al fine di controllare il massacro, I'ONU ha adottato la risoluzione 1246 dell'agosto 1999 per istituire una forza multinazionale per stabilizzare la situazione. Dopo il ritiro dall'Indonesia, TimorLeste è diventata beneficiaria di aiuti da parte della comunità internazionale, in quanto vi sono state perdite umane e materiali incalcolabili. II 20 maggio 2002, senza aiuti, l'indipendenza della Repubblica democratica di Timor-Leste è stata ripristinata, osservando il primo governo democraticamente eletto. ${ }^{[12]}$

L'interesse dell'Australia per Timor-Leste non è una novità. Gli australiani avevano informazioni che nel Mare di Timor-Leste c'era un sacco di gas e petrolio. Dalla scoperta nel 1960 che c'è stata ricchezza nel Mare di Timorese i governi stranieri hanno cercato di dominare l'isola. Negli anni '70, l'Australia è stato l'unico paese che 
ha riconosciuto che la metà occidentale dell'isola di Timor apparteneva all'Indonesia. Si scopre che nel 1970, una società australiana chiamata Woodside Petroleum ha trovato un'enorme riserva di gas nella regione conosciuta come The Sunrise e Troubadour Fields o Greater Sunrise. ${ }^{[13]}$ L'intenzione di negoziare per vantaggi territoriali è stata una delle ragioni per cui gli australiani hanno ignorato l'opinione negativa della comunità internazionale, che non era d'accordo con la condotta indonesiana di invadere la parte occidentale dell'isola di Timor. Interessati a fare profitti dalla scoperta della ricchezza nel Mare di Timor-Leste, Indonesia e Australia hanno firmato il trattato. Questo documento riguardava i confini marittimi tra i due paesi, ma l'Australia ha preso il territorio in cui si trovavano le riserve di gas. Insoddisfatto del trattato che ha firmato nel 1975 I'Indonesia ha invaso l'Australia per ottenere una percentuale sullo sfruttamento delle risorse di gas in quella regione. I timoresi non concordavano con il trattato che condivideva la ricchezza del loro territorio tra Indonesia e Australia, ma le due nazioni straniere coinvolte nell'accordo internazionale ignorarono la posizione del mondo, contrariamente al loro patto. II 20 maggio 2002, data del ripristino dell'indipendenza timor-leste, il paese ha cercato di impossessarsi del territorio che ne aveva diritto. Timor non apparteneva più all'Indonesia. I trattati che erano stati concordati prima dell'indipendenza non erano più preziosi. ${ }^{[14]}$

L'Australia, nel 2002, con l'obiettivo di rimanere dominare il territorio marittimo che sarebbe appartenuto a Timor Est, si è ritirata da tutte le procedure di frontiera vincolanti di cui faceva parte. Nel 2006 c'è stato un trattato tra l'Australia e Timor-Leste su alcuni adeguamenti marittimi nel Mare di Timor, un accordo che ha scontentato il timoreso perché serviva a evitare cambiamenti nei negoziati legali o azioni legali per cinquant'anni. È stato nel 2016 che le persone che si sentivano danneggiate dal Trattato hanno notificato agli australiani che avrebbe risolto il problema delle frontiere marittime con la conciliazione obbligatoria prevista dalla Convenzione UNITED NATIONS, I'articolo 298 e l'allegato V, che si occupa di Diritti del mare. ${ }^{[15]}$

Il dominio di Timor-Leste da parte di altre nazioni, in diversi periodi storici, lo ha gravemente danneggiato. Diverse riflessioni sullo sfruttamento dell'Indonesia e 
dell'Australia si fanno sentire nello sviluppo del popolo di Timorese in varie aree. Dal 2002, alla vigilia dell'indipendenza di Timor-Leste, gli australiani si sono ritirati da diversi trattati internazionali per evitare la perdita di sfruttamento del territorio non marittimo. Australiani e timoresi hanno territori separati per meno di 400 miglia nautiche. A causa della vicinanza tra le due nazioni, i loro territori marittimi devono essere definiti in modo peculiare e specifico, secondo il principio di equità. È essenziale approfondire la conoscenza della legge del mare e del petrolio per comprendere le ragioni di fatto e di diritto che legittimano la lotta del popolo di Timorese per il proprio territorio marittimo. ${ }^{[16]}$

\section{DESTRA DELLA SEA E OIL}

La Legge del Mare appartiene al diritto internazionale, che osserva la sovranità e la giurisdizione degli Stati, definendo l'estensione del loro dominio marittimo. Regola anche diversi altri argomenti come lo sfruttamento delle risorse esistenti sul letto e sul fondo marino, oltre alla conservazione e conservazione dell'ambiente marino. ${ }^{[17]}$ Una delle più grandi ricchezze che si trovano sul fondo del mare di Timorese è l'olio, che si distingue per essere un liquido nero viscoso e infiammabile, meno denso dell'acqua. Consiste in una miscela di idrocarburi, molecole composte da atomi di carbonio e idrogeno, così come molecole di zolfo, azoto, ossigeno e ioni metallici, e si trova in serbatoi sotterranei naturali. ${ }^{[18]}$

La Conferenza sui diritti marittimi si è svolta a Ginevra nel 1958. Nel 1969, è stato ratificato da quasi tutti i paesi del mondo. Si occupa di vari argomenti o argomenti che trattano con la stretta connessione nello spazio oceanico tra le nazioni. La Convenzione delle Nazioni Unite sui diritti del mare (UNCLOS) del 1982 è stata firmata a Montego Bay, in Giamaica, anche se è apparsa alla conferenza di Ginevra. In questo documento sono regole e principi del mare territoriale, aree con ${ }^{[19]}$ tigue, economiche e continentali. ${ }^{20]}$ Per quanto riguarda la sovranità dello Stato costiero sulle acque adiacenti, ${ }^{[21]}$ essa regola tutti i paesi che I'hanno adottata, accettata o ratificata. Esistono anche norme correlate in materia di gestione delle risorse marine e controllo dell'inquinamento. ${ }^{[22]}$ 
L'UNDP determina che il mare della costa ha 12 miglia nautiche, cioè $22 \mathrm{~km}$. Sul piano verticale, si estende allo spazio aereo, avendolo come limite. L'acqua di mare della superficie è limitata attraverso il contatto con il fondo dell'oceano, il suolo, così come tutto il sottosuolo è governato dal regime giuridico del mare territoriale. ${ }^{[23]}$ || diritto del mare agli Stati costieri è stato garantito lo sfruttamento economico esclusivo in un'aria di 200 miglia nautiche, ma questa regola non si applica agli Stati che si trovano a meno di 400 miglia di distanza dalle loro aree contigue. ${ }^{[24]}$

Figura 2 - Mare territoriale

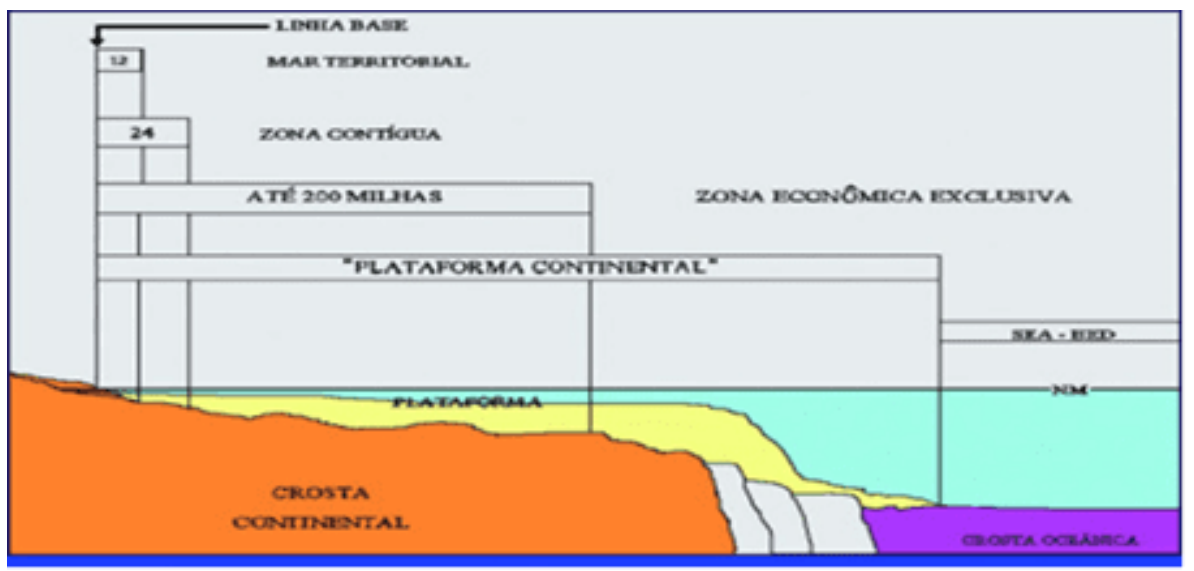

Fonte: GUSM-O (2018).

II Mare Territoriale è l'area situata tra le acque interne e l'Alto Mare. È la continuazione della sovranità di un paese costiero che supera il suo territorio e le sue acque, secondo gli articoli 2 e 3 dell'UNCLOS. ${ }^{[25]}$ Lo Stato esercita la sovranità sul suo mare territoriale, lo spazio aereo, così come il letto e il sottosuolo sotto il mare territoriale, secondo Art. da 02 a 32 della Convenzione sui diritti del mare. ${ }^{[2]}$ II confine esterno del mare territoriale di ogni nazione è di ventidue chilometri. Lo Stato esercita la sua giurisdizione sulle attività di interesse nazionale nell'area marittima contigua, che è fissata a 12 miglia. La Convenzione delle Nazioni Unite ha creato la zona economica esclusiva, l'EEE, per equilibrare gli interessi tra i paesi. Si tratta di un'area situata al di là del Mare Territoriale. Si riferisce alla zona vicino alla zona contigua e si estende fino a 200 miglia dalla costa. Lo stato ha il diritto di navigare, sorvolare, installare cavi e condotti marini, nonché di sfruttare i minerali presenti nel suolo e nel sottosuolo marino. 
Timor-Leste e l'Australia sono separate da meno di 400 miglia di distanza, e per questo motivo questa regola non risolve i conflitti oltre i confini tra le due nazioni. ${ }^{[27]}$

Tizi e australiani sono popoli appartenenti a stati con coste anteriori o situate adiacenti. Non soddisfano i limiti fissati dalla Convenzione delle Nazioni Unite per l'applicazione dei diritti del mare, legati alla sovranità dello Stato nel mare territoriale. La distanza dalla costa costiera tra i paesi è di 300 miglia. Questi paesi devono delimitare le frontiere marittime per accordo, come stabilito dalle regole del diritto internazionale. Le nazioni devono raggiungere una soluzione equa, equa ed equivalente. ${ }^{[28]}$

La Convenzione di Ginevra ha criteri per la delimitazione parallela. II metodo della linea mediana viene utilizzato in circostanze speciali per gli stati con schienali opposti. Regola un riadattamento della linea mediana tra i paesi. La Convenzione sulla legge del mare afferma che, in considerazione della mancanza di una distanza di 400 miglia per la separazione tra due paesi, dovrebbe essere utilizzato il principio di equidistanza. [29]

L'industria petrolifera è estremamente importante. La società di oggi dipende da essa e dai suoi derivati. È un combustibile fossile, una fonte di energia elettrica per la maggior parte dei paesi sviluppati e in via di sviluppo. È essenziale per la produzione di una gamma di prodotti come diesel, cherosene e benzina. È presente anche negli input e nell'industria petrolchimica, attraverso paraffina e nafta. Molti medicinali hanno nella loro composizione derivati del petrolio. È impossibile riflettere sugli attuali stampi della vita umana senza asfalto, plastica e aspirina. L'olio può essere osservato nei combustibili utilizzati per la locomozione delle persone. È contenuto in una moltitudine di sostanze chimiche e petrolchimiche fondamentali per lo sviluppo di una nazione. A causa della sua vasta applicabilità e perché è una fonte estenuante di energia, gli australiani hanno ignorato il diritto internazionale del mare per molti anni. Si sono ritirati dall'UNDP per impedire che il popolo di Timorese abbia diritto ai profitti sullo sfruttamento delle compagnie petrolifere Bayu Undan e Greater Sunrise. ${ }^{[30]}$

A causa dell'importanza del petrolio, l'Organizzazione dei paesi esportatori di petrolio (OPEC) è emersa a Vienna, in Austria, nel 1960. Le nazioni fondatrici erano la 
Repubblica islamica dell'Iran, I'Iraq, il Kuwait, I'Arabia Saudita e il Venezuela. L'obiettivo dell'Opec è quello di stabilire una politica comune per il petrolio, proteggendo i redditi dei paesi produttori. Prima della creazione di questa organizzazione, i paesi esportatori di petrolio che detenevano la maggior parte delle riserve petrolifere del tempo hanno beneficiato poco. Le grandi società regolavano il mercato petrolifero internazionale. II valore dei combustibili fossili versati ai paesi produttori e per la rivendita al consumatore finale era controllato dalle compagnie petrolifere. Le compagnie americane Exxon, Texaco, Amoco e Chevro, così come la Dutch Royal Dutch Shell e la British Petroleum furono chiamate Seven Sisters. Hanno effettuato l'esplorazione, la raffinazione, il trasporto e la rivendita di petrolio, ma solo una piccola parte dei frutti dell'estrazione era destinata agli Stati produttori. Attualmente, le Nazioni Unite osservano anche le azioni delle compagnie petrolifere in modo che possa verificarsi un accordo appropriato tra gli Stati produttori e le società di estrazione del petrolio. ${ }^{[31]}$

Timor-Leste è stato esplorato per decenni dall'Australia. Questo comportamento è stato sostanzialmente guidato da ragioni economiche, a causa dell'interesse per la ricchezza del territorio marino di stanero. Non è nuovo che l'industria petrolifera sia motivata a rimuovere l'Australia dalla comunità internazionale. Gli australiani non sono stati disposti ad affrontare questioni relative ai diritti del mare. L'interesse per i profitti derivanti dall'estrazione di petrolio e gas naturale dalla regione appartenente a TimorLeste ha impedito a tale regione di raggiungere per molti anni un accordo tra le due nazioni. ${ }^{[32]}$

Timoresi è stata danneggiata dall'Australia dall'indipendenza nel 2002. Tutte le riserve di petrolio e gas si trovano sul lato timorese della linea mediana, cioè più vicino a Timor-Leste che al territorio australiano. L'Australia non riconosce più la giurisdizione della Corte internazionale di giustizia e della Convenzione delle Nazioni Unite sul diritto del mare per delimitare i suoi confini sulla linea mediana con Timor-Leste mesi prima della sua indipendenza. L'allontanamento dell'Australia dall'UNDP aveva l'intenzione di evitare la perdita o la riduzione dello spazio territoriale marittimo, in quanto apparteneva al diritto ai timoresi. La Convenzione delle Nazioni Unite sui diritti del mare 
afferma che ogni paese deve delimitare come una zona economica esclusiva 200 miglia nautiche, dal limite esterno del suo mare territoriale, 12 miglia dalla costa. L'UNDP si occupa anche delle grandi profondità del mare che sono conosciute come Area o zona $A$, secondo l'arte. 1. Questa zona è composta da letti marini e oceanici e il loro sottosuolo. ${ }^{[33]}$

Figura 3 - Linea di demarcazione del confine Australia

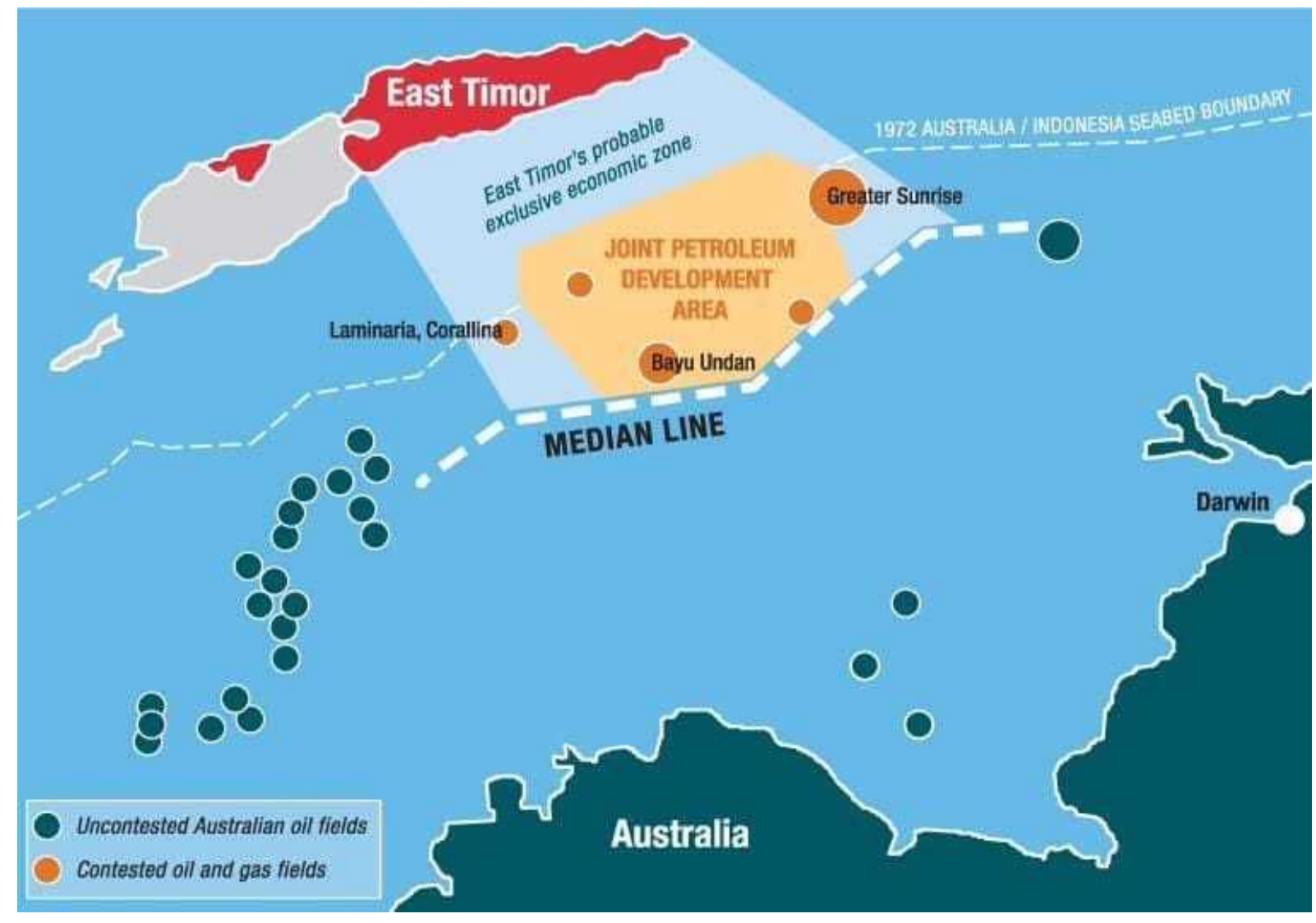

Fonte: TIMOR SEA JUSTICE (2016). [34]

I principi contenuti nella Convenzione delle Nazioni Unite sui diritti del mare affermano che il confine marittimo tra i due paesi coinvolti dovrebbe essere definito dalla linea equidistante, cioè dalla linea mediana. La demarcazione dei confini tra l'Australia e Timor-Leste attraverso il principio di equidistanza include territorio timoreso con l'intera zona di esplorazione del pozzo della compagnia petrolifera Bayu Undan. II giacimento petrolifero della Grande Alba si trova nella cosiddetta area La zona di cooperazione e, secondo UNCLOS, questo territorio appartiene anche al popolo di Timorese. ${ }^{[35]}$ 
Il petrolio è una ricchezza che si trova in abbondanza a Timor-Leste. La linea mediata deve essere tracciata per definire lo spazio marittimo tra due paesi che separano per meno di 400 miglia nautiche. I serbatoi di gas naturale e petrolio sono stati trovati dalle aziende australiane a Timor-Leste, prima della loro indipendenza. Per questo motivo, gli australiani hanno avuto molta resistenza a un trattato definitivo sul diritto allo sfruttamento del Mare di Timorese. Le difficoltà incontrate da queste persone per ottenere i loro diritti marittimi sono questioni importanti. L'analisi dei dettagli che hanno preceduto il trattato definitivo realizzato nel 2018, che si occupa dei confini di Timorese, è essenziale per comprendere la proporzione di sviluppi che questo patto intende. [36]

\section{TRATTATO TRA AUSTRALIA E TIMOR-LESTE}

Nel 2002, Timor-Leste ha ottenuto l'indipendenza dall'Indonesia, ma da quell'anno, i confini marittimi definitivi di Timor non sono stati delimitati. Gli australiani, per molti anni, non hanno alcun interesse a trattare con i confini marittimi timor-leste, rispettando gli standard del diritto internazionale. Essi intendevano rimanere utilizzando le risorse del mare che non le appartenevano. Sebbene abbiano causato danni allo sviluppo di Timor Est in diverse zone per lungo tempo, il patto non prevede il risarcimento dei danni causati dal 2002. ${ }^{[37]}$

Il popolo di Timorese non sarà rimborsato per i danni causati alla loro evoluzione nei settori dell'istruzione, sociale, economico, politico, tra gli altri che richiedono risorse finanziarie minime. Ma quando il patto è stato realizzato, è stato osservato che Timor Est è stato gravemente danneggiato nel corso degli anni dall'Australia. ${ }^{[38]} \mathrm{Al}$ fine di incoraggiare lo sviluppo, industriale, tecnologico ed economico di famorese, si è convenuto che l'azienda che avrebbe effettuato l'esplorazione della Grande Alba avrebbe dovuto assumere i cittadini di Timor Est, facilitando loro la formazione incoraggiare lo sviluppo dei loro studi, nonché avrebbe il dovere di adottare il paese come prima opzione per l'acquisizione di beni e servizi. ${ }^{[39]}$

Al fine di evitare perdite economiche, l'Australia si è ritirata da diversi trattati internazionali di cui era parte mesi prima dell'indipendenza di Timor-Leste. ${ }^{[40]} \mathrm{Al}$ fine di evitare che uno dei due paesi non osservi il patto in futuro, le nazioni hanno stabilito 
che il trattato non sarebbe stato soggetto a un diritto unilaterale di denuncia, ritiro o sospensione. Nel testo del documento è stato chiarito che tutte le clausole espresse nel trattato e negli allegati fanno parte del patto e non possono essere ignorate 0 evidenziate. Di fronte a possibili controversie future, si è convenuto che potrebbe essere risolto con l'aiuto dei membri della Commissione di conciliazione che hanno mediato il patto tra le due nazioni nel 2018. Tuttavia, nella permanenza del disaccordo sulle questioni, le questioni affrontate con l'intervento dei membri del comitato di conciliazione potrebbero essere portate dinanzi al tribunale arbitrale, e tale seconda decisione avrebbe un effetto vincolante. ${ }^{[41]}$

II 6 marzo 2018 ha avuto luogo l'accordo tra Timor-Leste e l'Australia. Questo patto delimita il bacino continentale. In questo documento, i confini mediani e laterali sono stati delimitati ad est e ad ovest della vecchia zona conosciuta come Timor Gap, secondo l'arte. Primo trattato sulle frontiere marittime di Timor-leste. ${ }^{[42]} \mathrm{Da}$ questo nuovo contesto, Timor-Leste è autorizzata a svolgere lo sfruttamento delle risorse marine del suolo come il petrolio e il gas naturale, oltre a fornire i benefici ai diritti della zona economica esclusiva. Questa nazione ha anche guadagnato il diritto di sfruttare altre risorse marittime come la pesca, consentendo maggiori risorse finanziarie per il paese. ${ }^{[43]}$

Figura 4 - Trattato dell'Australia e Timor-Leste sui confini di Timor

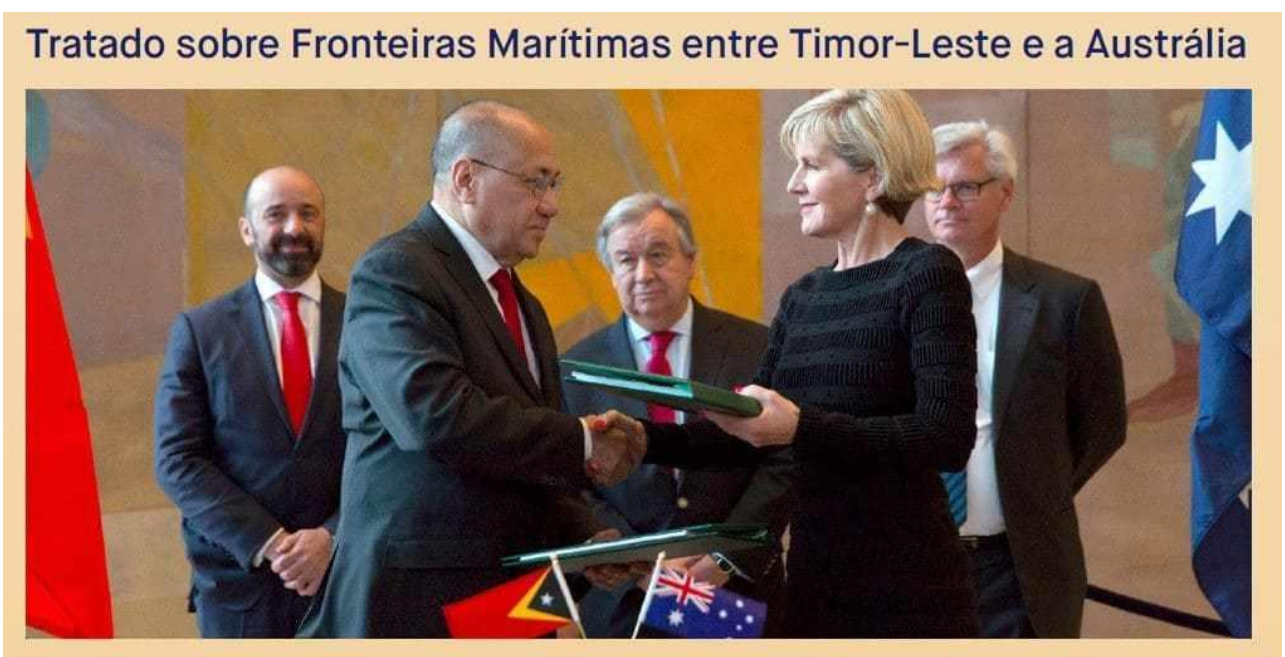

Fonte: Timor-Leste (AUSTRALIA, 2018). [44] 
Timor-Leste e l'Australia hanno allentato i loro interessi per risolvere i disaccordi che impedivano loro di concordare. Nell'area territoriale marittima in cui si trova la Grande Alba, si è convenuto che sarebbe appartenuta a ciascuno dei paesi coinvolti nell'accordo per metà dei risultati del funzionamento di tale regione territoriale marittima. I campi bayu-undan, Buffalo e Kitan hanno completamente integrato il territorio timoreso. ${ }^{[45]}$

I modi in cui le entrate sarebbero ripartiti tra Timor Est e l'Australia non sono stati definiti, in quanto dipenderebbero da diversi fattori. Quanto maggiore è la capacità di sviluppare tecniche e applicarle, tanto maggiori sarebbero i loro profitti. Secondo i modi di esplorare i campi della Grande Alba, i risultati sarebbero condivisi. Nell'anno in cui si è verificato il patto, si è convenuto che le entrate dei giacimenti petroliferi sarebbero appartenute al 30\% all'Australia e al $70 \%$ per Timor Est se ci fosse stato un aumento dell'esplorazione attraverso lo sviluppo di gasdotti per il popolo di Timor. Si prevedeva inoltre che, nel caso in cui i campi di Greater Sunrise saranno sviluppati attraverso un Pipeline to Australia, le percentuali sarebbero state modificate, appartenenti al $20 \%$ per gli australiani e all' $80 \%$ per Timorese. ${ }^{[46]}$

Nel trattato definitivo sui confini, secondo l'art. 12 del documento giuridico, è stato definito che l'esplorazione del gas e del petrolio nella regione del campo della Greater Sunrise dipenderà dalla definizione tra le parti sul piano di sviluppo. Si scopre che dopo l'inizio delle attività di esplorazione della regione marittima che avrebbe condiviso i ricavi, la società appaltatrice avrebbe presentato alla giurisdizione esclusiva del paese in cui si trova. Sarebbe istituito il Consiglio di sorveglianza di due rappresentanti di Timor Est e un rappresentante australiano. Al fine di trovare soluzioni al contenzioso, ci sarebbe un comitato indipendente di risoluzione dei conflitti per le decisioni sulle strategie adottate per l'esplorazione del campo petrolifero di cui sopra. ${ }^{[47]}$

Per decenni, I'Australia ha dimostrato la sua intenzione di trarre profitti dallo sfruttamento dei giacimenti petroliferi in pieno. È stato necessario un intervento internazionale per ridurre il conflitto sui confini tra Timoresi e gli australiani. II Trattato definitivo sulle frontiere si occupa della linea mediana e dei limiti laterali sul territorio di Timor-Leste e condivide le entrate di una regione petrolifera tra i paesi interessati. Ma 
questo patto non estingue la possibilità di futuri conflitti sui profitti provenienti dai campi della Greater Sunrise. Molte domande erano in attesa di chiarimenti, che richiedevano conflitti concreti per l'adozione di posizioni. Di fronte a questa nuova situazione, il popolo di Timorese dovrà affrontare diversi ostacoli in modo che possano godere di tutte le possibilità per il loro sviluppo in vari settori. ${ }^{[48]}$

\section{Gli OSTACOLI PER LO SVILUPPO DI TIMOR-LESTE}

Timor-Leste è uno stato marittimo e non solo il petrolio. Ci sono diversi settori che possono contribuire all'evoluzione della nazione. Per molti anni è stato esplorato da Portogallo, Indonesia e Australia. Sono state investite poche risorse nell'istruzione e nelle infrastrutture. La nazione è stata danneggiata nello sviluppo di diverse aree a causa dell'abbandono dell'Indonesia. Ci sono pochi professionisti con competenze tecniche, politiche, legali e amministrative, perché durante il periodo di dominazione della gente, solo i professionisti stranieri sono stati assunti per svolgere attività in questi settori. ${ }^{[49]}$

Figura 5 - Studenti di una scuola della periferia di Timorese

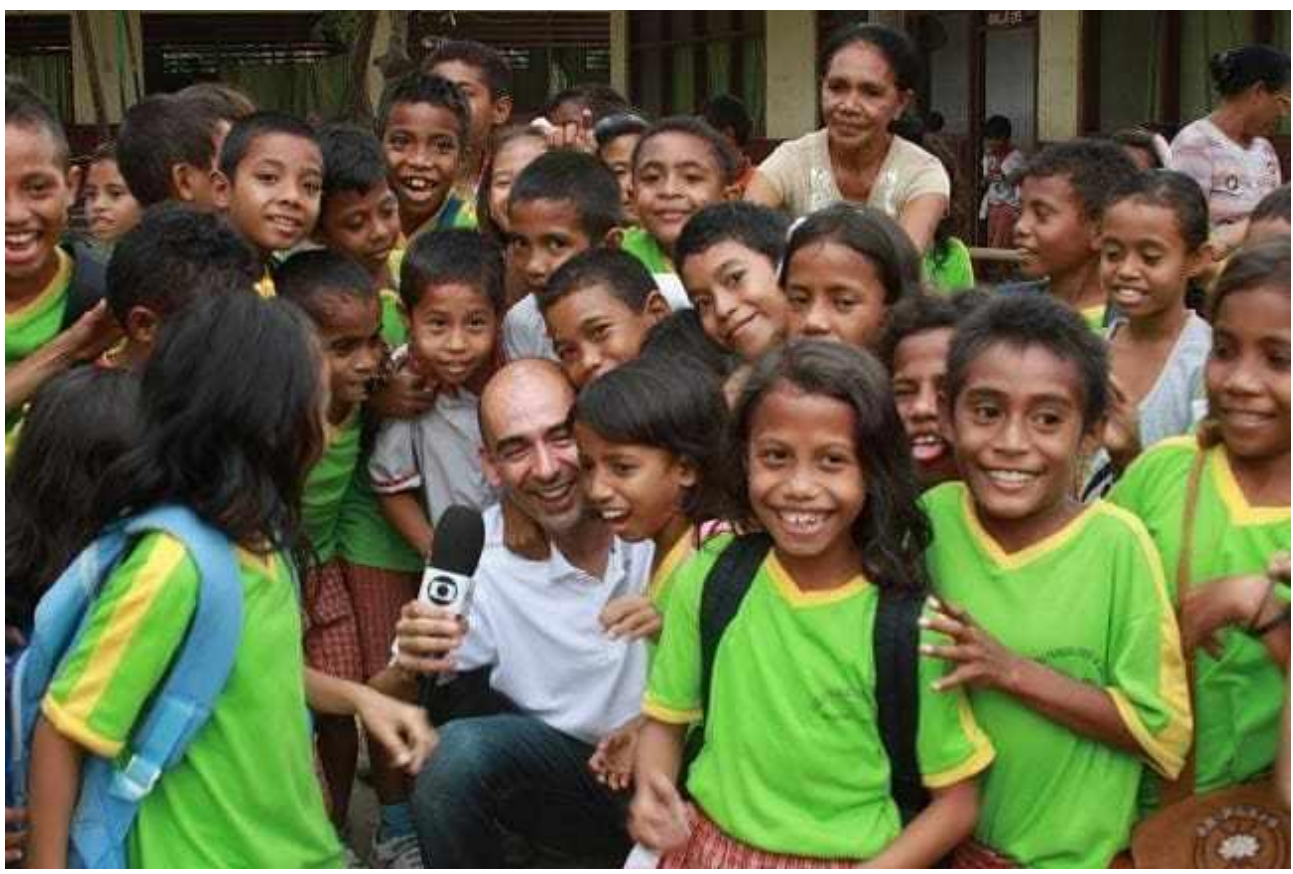

Fonte: Partners for Peace (2011). [50] 
Dal 2011, Timor-Leste ha un piano di sviluppo strategico che include l'evoluzione del capitale umano, delle infrastrutture, dell'economia, della politica e dello spazio amministrativo. L'obiettivo del governo di Timorese è quello di raggiungere gli obiettivi fissati nel piano di sviluppo entro il 2030. Le risorse derivanti dai diritti di sfruttamento del suo territorio marittimo porteranno maggiore rapidità nell'attuazione degli obiettivi proposti nel piano di sviluppo di Timorese. II patto australiano sui confini definitivi genererà certamente diverse situazioni particolari e senza precedenti. Conoscenze specifiche sui metodi di esplorazione del petrolio e del gas naturale saranno essenziali per capire quali alternative porteranno il maggior numero di profitti al paese. Dopo la ratifica del trattato, sono state stabilite molte norme in modo che i stancamentesi potessero godere dei loro diritti, ma avranno bisogno di professionisti qualificati in vari settori. E una delle principali sfide che Timor-Leste deve affrontare è la breve esperienza nella pubblica amministrazione, con grandi volumi di entrate finanziarie, che si moltiplicheranno a causa del trattato definitivo sui suoi confini. ${ }^{[51]}$

Nel documento del trattato sono state stabilite norme che coinvolgono grandi società di esploratori internazionali. Le compagnie petrolifere dovrebbero contribuire allo sviluppo di timor-leste, preferibilmente assumendo professionisti, servizi e prodotti nel paese. Timoresi non si riferirà non solo ai rappresentanti del governo australiano, ma con la somma di iniziative private con esperienza. I rappresentanti stranieri del governo australiano o di aziende private di varie nazioni possono avere una vasta capacità di negoziare e trattare con Timoresi inesperti. Timor-Leste ha professionisti con poca abilità tecnica, ma questa situazione non dovrebbe compromettere lo sviluppo. La missione di questi imprenditori sarà quella di assistere nell'educazione e nell'insegnamento delle competenze e delle conoscenze necessarie ai lavoratori per svolgere compiti relativi alle attività di esplorazione. L'aumento delle risorse finanziarie contribuirà alla trasformazione dei settori, tecnici, amministrativi, politici, giuridici, tra gli altri, facilitando la trasposizione degli ostacoli allo sviluppo della nazione. ${ }^{[52]}$

L'esplorazione del gas e del petrolio richiederà che il potere legislativo di Timor Leste sia consapevole dell'emergere di nuovi fatti derivanti da questa attività. Le agenzie statali dovranno trovare risposte rapide alle divergenze tra lo Stato, i rappresentanti 
delle società di sfruttamento e l'Australia. Anche se la società appaltata ha l'obbligo di sottomettersi alla giurisdizione esclusiva del paese in cui si trova, l'esperienza e la capacità dei rappresentanti del settore privato di manipolare i negoziati dovrebbero essere considerati. È innegabile che il governo di Timor Est adotti posizioni che contribuiscono al progresso in varie aree del paese, ma è un dato di fatto che i poteri esecutivi, legislativi e giudiziari di stancazione sono ancora in via di sviluppo, perché è un paese molto giovane. ${ }^{[53]}$

Timor-Leste ha una vasta esperienza nella lotta, come il popolo organizzato contro l'invasione dell'Indonesia e ottenuto la loro indipendenza. ${ }^{[54]}$ Attualmente, avanza alla ricerca di istruzione, infrastrutture, economia, politica e un'adeguata gestione amministrativa per i cittadini. Nel patto sulle frontiere definitive è stata riconosciuta la situazione di fragilità di Timor Est. E i meccanismi per aiutare nell'evoluzione del paese sono stati positivi nell'accordo. ${ }^{[55]}$

II governo di Timor-Leste avrà compiti complessi e sarà necessario un settore giuridico e amministrativo pronto ad agire in nuove attività. I rapporti e i piani di sviluppo sull'esplorazione dei campi di Bayu-Undan, Buffalo e Kitan dovranno essere pubblicati durante il periodo di transizione di esplorazione di quella regione. Questo dovere richiederà conoscenze tecniche specifiche sull'esplorazione del petrolio e del gas. ${ }^{[56]}$ Molti settori sono legati allo sviluppo di una nazione. Le politiche di investimento in settori che dispongono di fonti di sfruttamento rinnovabile devono essere osservate dal governo di Timor-Leste. Dovrebbero essere prese in considerazione le disposizioni del Patto sulle frontiere di Timoresi, relative all'obbligo delle imprese di contribuire all'evoluzione dei vari settori. Lo Stato dovrà lottare per lo sviluppo nella stessa misura in cui dovrebbe richiedere un'iniziativa privata per contribuire all'evoluzione del paese. ${ }^{[57]}$

La crescita economica è fondamentale per il progresso delle infrastrutture, della politica e dell'amministrazione. L'ascesa economica di Timor-Leste è legata ai settori dell'agricoltura, del turismo e del mare e non solo all'esplorazione di petrolio e gas. L'agricoltura è essenziale per Timor-Leste, così come ha grande rilevanza per molte altre nazioni. Da esso è che il cibo viene prodotto. I prodotti primari dell'agricoltura 
possono essere utilizzati dalle industrie, dal commercio e dal settore dei servizi. Possono diventare la base per il mantenimento dell'economia nazionale e internazionale. ${ }^{[58]}$

L'esplorazione dell'economia marittima è anche cruciale per l'evoluzione della nazione di Timorese. Molte persone dipendono dal mare e si sostengono con le risorse generate dalla pesca e dalla raccolta delle specie marine. Si scopre che, nel territorio marittimo di quel paese, c'è un naturale passaggio di pesce. E attraverso gli sviluppi portati dal patto definitivo sulle frontiere, diverse specie di pesci potrebbero essere sfruttate dalle industrie nazionali. Oltre all'aumento delle entrate per l'emergere di industrie interessate alle molte specie di scuole che quella regione ha, questa nazione si trova nel Triangolo dei Coralli. Si tratta di un patrimonio culturale che può essere apprezzato dal turismo, così come può servire come un interesse per la ricerca scientifica. Le caratteristiche di cui sopra possono generare l'aumento diretto e indiretto delle risorse per lo Stato, a condizione che si verifichino adeguate misure amministrative. ${ }^{[59]}$

Anche le infrastrutture dei porti e degli aeroporti di Timoresi sono fondamentali per la crescita dei paesi. I parametri delle costruzioni strategiche devono monitorare le esigenze di navigazione dello scenario globale che il territorio di Timorese possa transitare attraverso il territorio di Timorese. I porti di Dili, Oe-cusse, Hera, Caravela e Com hanno attualmente caratteristiche inadeguate, che richiedono che gli investimenti siano considerati centri industriali. Questa evoluzione creerà opportunità per TimorLeste di diventare un transito di merci petrolifere, oltre a renderla una nazione in possesso di un grande hub industriale che collega l'Oceano Pacifico e l'Oceano Indiano. ${ }^{[60]}$

Timor-Leste deve continuare ad avanzare in molti settori. I settori agricolo, petrolifero, marittimo, turistico, educativo, giuridico, politico ed economico necessitano di trasformazioni. Esiste già un piano di politica di sviluppo creato nel 2011. Tuttavia, è necessario adattarlo alla nuova realtà in cui si trova il paese, dopo i cambiamenti che si sono verificati dalla definizione dei suoi confini marittimi finali. ${ }^{[61]}$ Occorrerà ripercorrere strategie specifiche per ciascun settore a livello nazionale interno. E, sulla 
base di questi nuovi obiettivi, dovrebbero essere adottate le posizioni con le società di esplorazione del petrolio e del gas, così come tutte le decisioni dello Stato saranno al fine di raggiungere questi obiettivi di crescita. ${ }^{[62]}$

\section{CONCLUSIONE}

Timor-Leste si trova nel sud-est asiatico ed è un paese molto giovane, che ha ottenuto la sua indipendenza nel 2002. È il vicino dell'Indonesia, che si trova a nord, e a sud è I'Australia. Ha una nazione caratterizzata dalla sua forza e lotta. Questo guerriero e persone sofferenti comunicano attraverso la lingua portoghese e Tetum, ufficialmente, ma nel territorio ci sono più di 14 lingue native. Fu colonizzata dal Portogallo e invasa dall'Indonesia nel 1975. Ha un tesoro situato nel suo territorio marittimo, a causa del petrolio e del gas naturale che nel mare di Timorese si incontrano. Queste ricchezze di Timor-Leste furono oggetto di una battaglia con l'Australia. II disaccordo tra australiani e timoresi iniziò mesi prima dell'indipendenza di Timor-Leste dell'Indonesia. L'Australia non aveva alcun interesse a negoziare, come previsto dal diritto internazionale del mare, per evitare la perdita di entrate derivanti dal mare di Timorese. Ma nel 2018 si è tenuto con gli australiani un trattato definitivo sui confini di Timor-Leste. Questo è stato il momento storico in cui la nazione ha ottenuto il riconoscimento del diritto di godere del suo mare territoriale.

La delicata situazione economica del paese è stata riconosciuta nel trattato di frontiera definitivo di Timor-Leste. La scarsità di risorse economiche è legata allo sfruttamento di questa nazione in modo devastante. II Portogallo ha investito poco durante il periodo di oltre 400 anni in cui questo paese è stata la sua colonia. Dopo essere stato liberato dalla dominazione portoghese, ebbe un nuovo attacco a Timor-Leste. Per più di ventiquattro anni, i timoresi soffrirono dell'occupazione dell'Indonesia, ma furono fatti piccoli investimenti. Tuttavia, dal periodo in cui il popolo di Timoresi ha mostrato interesse a combattere per la loro indipendenza, tutto è stato distrutto dal popolo indonesiano. Nella fase iniziale dell'occupazione di Timor-Leste da parte dell'Indonesia, la comunità internazionale ha contribuito con l'aiuto alla ricostruzione di base del paese. 
L'indipendenza dei timoresi non ha dato loro il diritto immediato di impossessarsi della ricchezza marittima che apparteneva loro, a causa del fatto che erano sul loro territorio. Accordi interinali sulle frontiere, patti che danneggiavano il timoreso si sono svolti con l'Australia prima del 2018. Dal 2002, Timor-Leste rivendica la proprietà del suo territorio marittimo, come previsto dalle norme di diritto internazionale, UNCLOS/1982. Si scopre che pochi mesi prima che Timor-Leste otasse la sua indipendenza, I'Australia si ritirò da diversi patti internazionali.

Poiché i territori marittimi di Timor-Leste e dell'Australia sono separati da una distanza di meno di 400 miglia nautiche, a breve distanza tra i territori delle due nazioni, la linea di delimitazione delle frontiere adottata è stata definita dallo standard di equidistanza e linea mediana. Questo è il modello previsto dalla Convenzione delle Nazioni Unite per i diritti del mare del 1982, utilizzato per risolvere i conflitti che si sono occupati di territori che si allontanano per meno di 400 miglia nautiche.

Gli australiani hanno evitato di raggiungere un accordo con timoresi sulle frontiere definitive, in conformità con le regole della Convenzione delle Nazioni Unite sui diritti del mare per molti anni. Lo scopo di questa posizione era quello di mancare di rispetto al modo di delimitare le frontiere marittime, che fornisce come standard l'adozione della linea mediana e l'equidistanza in specifici conflitti sulla definizione delle frontiere. La ragione della rimozione dell'Australia dalla comunità internazionale è legata all'estensione del profitto dallo sfruttamento del mare che appartiene al popolo di Timorese.

Dopo diversi tentativi di accordarsi definitivamente sui confini di Timoresi con l'Australia, il paese è riuscito. Chiaramente e nello specifico, in un documento che contiene più di 11 articoli, i confini marittimi bilaterali permanenti sono stati affrontati lungo una linea essenzialmente equidistante tra le due nazioni. Timor-Leste aveva $i$ suoi confini delimitati come previsto dalla Convenzione delle Nazioni Unite sulla legge del mare. Nel trattato è stato concordato che esiste un legame indivisibile tra il confine marittimo tra Timor-Leste e l'Australia. Si è riconosciuto l'esistenza di un'area marittima appartenente alle due nazioni coinvolte nel patto. 
Durante la preparazione del documento, le parti hanno riunito le entrate derivanti dallo sfruttamento di maggiori campi di alba e positive nel testo del patto internazionale la necessità di elaborare norme riguardanti lo sfruttamento dei campi della regione appartenenti ai due Paesi. È stato accertato che le norme concordate nel trattato o nei suoi allegati non possono essere parzialmente inosservate da nessuna delle parti coinvolte. È stato inoltre regolamentato sulla necessità di creare piani di esplorazione della ricchezza marittima prima dell'inizio delle procedure, nonché che si è anche convenuto che le compagnie petrolifere avrebbero bisogno di acquistare prodotti e servizi preferibilmente dal popolo di Timorese.

Il patto di frontiera definitivo di Timor Est non è solo un accordo comune tra i governi di due nazioni. Questo patto timoreso e australiano copre anche gli obblighi di iniziativa privata. Sono stati assegnati dazi alle imprese che effettueranno l'esplorazione di petrolio e gas nella regione dei settori della Greater Sunrise. Essi dovrebbero contribuire allo sviluppo di Timor-Leste.

Molti ostacoli hanno dovuto essere eliminati in modo che Timor-Leste possa svilupparsi ampiamente, pur avendo ottenuto il diritto di esplorare il suo territorio marittimo. II petrolio e il gas sono risorse non rinnovabili, cioè sono fonti di reddito con una cessazione stimata. Ma sono fonti di reddito immediato e redditizio. Lo sviluppo dell'istruzione, delle infrastrutture, dell'agricoltura, del turismo e dell'erogazione dei servizi è essenziale per costruire una base di reddito solida e rinnovabile a medio e lungo termine. II paese si trova ad affrontare diversi ostacoli, come poche infrastrutture e basso livello di istruzione, ma è in forte crescita dal 2002.

In un mondo capitalista, è innegabile che le risorse finanziarie sono fattori determinanti per l'ampia evoluzione di una nazione. L'aumento delle entrate derivanti dall'esplorazione di petrolio e gas aumenterà le entrate finanziarie, un fatto che contribuirà all'evoluzione dell'istruzione e degli investimenti nelle infrastrutture dei porti e degli aeroporti di Timor-Leste. Se, dinanzi al trattato sulle frontiere definitive, l'istruzione, le infrastrutture, l'agricoltura e la pesca industriale sono stati ostacoli allo sviluppo di FAmorese, si prevede che, dopo il patto definitivo con I'Australia, questa realtà sarà modificata. 


\section{RIFERIMENTI}

BRASIL. Decreto no 99.165, de 12 de março de 1990. Convenção das Nações Unidas sobre o Direito do Mar. Brasília: Planalto, 1990. Disponível em: https://www2.camara.leg.br/legin/fed/decret/1990/decreto-99165-12-marco-1990328535-publicacaooriginal-1-pe.html. Acesso em 15 abr.19.

DEL'OMO, Florisbal de Souza. Curso de direito internacional público. Rio de Janeiro: Forense, 2006.

GIBERTONI, Carla Adriana Comitre. Teoria e prática do direito marítimo. Rio de Janeiro: Renovar, 1998.

GOMES, Danaciano. Timor Leste: A economia do mar: um contributo para desenvolvimento sustentável. Aveiro: Mare Liberum, 2016.

GUSMÃO, Kay Raia Xanana. Breve história do mar do Timor. In: GOVERNO DO ESTADO DO TIMOR LESTE. Novas Fronteiras: conciliação histórica das fronteiras marítimas no mar do timor. Dili: Gabinete das Fronteiras marítimas, 2018.

MARCONI, Maria de Andrade, Lakatos, Eva, Maria. Fundamentos da metodologia cientifica. São Paulo: Atlas. 2003.

MARITIME BOUNDARY OFFICE. New frontiers: Timor-Leste's historic conciliation on maritime boundaries in the timor sea. [S. I.], 2015. Disponível em: http://www.gfm.t//wp-content/uploads/2015/11/Website-map-of-Timor-Sea.pdf. Acesso em: 04 maio 2019.

MATTOS, Adherbal. Meira. O novo direito do mar. Rio de Janeiro: Renovar. 1996.

PEREIRA, Eliana Sofia da Silva. Contributo crítico para a compreensão do regime do Mar de Timor à luz do Direito Internacional. 2013. 87 f. Dissertação. (Mestrado em Ciências Jurídicas Internacionais) Faculdade de direito- Universidade Nova de Lisboa, 2013. p.14.

Disponível em: 
https://run.unl.pt/bitstream/10362/17481/1/Pereira_2013.pdf. Acesso em: 01 abr. 2019.

REPÚBLICA DEMOCRÁTICA DE TIMOR-LESTE. Ministério das finanças. Orçamento geral do Estado 2018. Díli: Gabinete Ministerial, 2018. p. 7. Disponível em: https://www.mof.gov.tl/wp-content/uploads/2018/08/BB5_Port.pdf. Acesso em: 15 maio 2019.

REPUBLICA DEMOCRÁTICA DO TIMOR LESTE. História do Timor Leste. Governo do Timor Leste, Dili, [s. d.]. Disponível em: http://timor-leste.gov.tl/?p=29\&lang=pt. acesso em: 05 maio 2019.

REZEK, José Francisco. Direito internacional público: curso elementar. 10. ed. rev. e atual. São Paulo: Saraiva, 2005.

RIBEIRO, Marilda, Rosado de Sá. Direito do petróleo. 3. ed. rev. atual. e ampl. Rio de Janeiro: Renovar, 2018.

TIMOR LESTE; AUSTRÁLIA. Tratado sobre fronteiras marítimas entre Timor Leste e Austrália que estabelece as respectivas fronteiras do mar do Timor Leste. Nova York: [s.n.], 2018. p. 1. Disponível em: http://www.gfm.t//wpcontent/uploads/2018/03/Port-Timos-Sea-Maritime-BoundaryTreaty_Portuguese.pdf. Acesso em:04 maio 2018.

TIMOR SEA JUSTICE. All of the contested oil \& gas fields are on EastTimor's side of the median line ie closer to \#Timor than Australia. Dili, 01 mar. 2016. Tiwitte: @timor sea justice. Disponível em: https://twitter.com/TimorSeaJustice/status/704895304701763584. Acesso em: 25 maio. 2019.

ZANELLA, T. V. Curso de direito do mar. Curitiba: Juruá, 2013. 


\section{APÊNDICE - REFERENCIAS DE NOTA DE RODAPÉ}

3. GOMES, Danaciano. Timor-Leste: A economia do mar: um contributo para desenvolvimento sustentável. Aveiro: Mare Liberum, 2016.

4. GUSMÃO, Kay Raia Xanana. Breve história do mar do Timor. In: GOVERNO DO ESTADO DO TIMOR LESTE. Novas Fronteiras: conciliação histórica das fronteiras marítimas no mar do timor. Dili: Gabinete das Fronteiras marítimas, 2018. p. 6.

5. RIBEIRO, Marilda, Rosado de Sá. Direito do Petróleo. 3. ed. rev. atual. e ampl. Rio de Janeiro: Renovar, 2018.

6. RIBEIRO, Marilda, Rosado de Sá. Direito do Petróleo. 3. ed. rev. atual e ampl. Rio de Janeiro Renovar, 2018.

7. GOMES, Danaciano. Timor Leste: a economia do mar: um contributo para desenvolvimento sustentável. Aveiro: Mare Liberum, 2016.

8. MARCONI, Maria de Andrade, Lakatos, Eva, Maria. Fundamentos da metodologia cientifica. São Paulo: Atlas. 2003.

9. GUSMÃO, Kay Raia Xanana. Breve história do mar do Timor. In: GOVERNO DO ESTADO DO TIMOR LESTE. Novas Fronteiras: conciliação histórica das fronteiras marítimas no mar do timor. Dili: Gabinete das Fronteiras marítimas, 2018. p. 7-8.

10. MARITIME BOUNDARY OFFICE. New frontiers: Timor-Leste's historic conciliation on maritime boundaries in the timor sea. [S. I.], 2015. Disponível em: http://www.gfm.tl/wp-content/uploads/2015/11/Website-map-of-Timor-Sea.pdf. Acesso em: 04 maio 2019.

11. GOMES, Danaciano. Timor Leste: A economia do mar: um contributo para desenvolvimento sustentável. Aveiro: Mare Liberum, 2016. p. 35-36.

12. GOMES, Danaciano. Timor Leste: A economia do mar: um contributo para desenvolvimento sustentável. Aveiro: Mare Liberum, 2016. p. 37 e 38 
13. PEREIRA, Eliana Sofia da Silva. Contributo crítico para a compreensão do regime do Mar de Timor à luz do Direito Internacional. 2013. 87 f. Dissertação. (Mestrado em Ciências Jurídicas Internacionais) Faculdade de direito- Universidade Nova de Lisboa, 2013. p.14. Disponível em: https://run.unl.pt/bitstream/10362/17481/1/Pereira_2013.pdf. Acesso em: 01 abr. 2019.

14. PEREIRA, Eliana Sofia da Silva. Contributo crítico para a compreensão do regime do Mar de Timor à luz do Direito Internacional. 2013. 87 f. Dissertação. (Mestrado em Ciências Jurídicas Internacionais) Faculdade de direito- Universidade Nova de Lisboa, 2013. p.14. Disponível em: https://run.unl.pt/bitstream/10362/17481/1/Pereira_2013.pdf. Acesso em: 01 abr. 2019.

15. TIMOR LESTE; AUSTRÁLIA. Tratado sobre Fronteiras Marítimas entre Timor Leste e Austrália que estabelece as respectivas fronteiras do mar do Timor Leste. Nova York: [s.n.], 2018. p. 1. Disponível em: http://www.gfm.t//wpcontent/uploads/2018/03/Port-Timos-Sea-Maritime-Boundary-

Treaty_Portuguese.pdf. Acesso em:04 maio 2018.

16. PEREIRA, Eliana Sofia da Silva. Contributo crítico para a compreensão do regime do Mar de Timor à luz do Direito Internacional. 2013. 87 f. Dissertação. (Mestrado em Ciências Jurídicas Internacionais) Faculdade de direito- Universidade Nova de Lisboa, 2013. p.14. Disponível em: https://run.unl.pt/bitstream/10362/17481/1/Pereira_2013.pdf. Acesso em: 01 abr. 2019.

17. MATTOS, Adherbal. Meira. O novo Direito do Mar. Rio de Janeiro: Renovar. 1996. p. 04

18. RIBEIRO, Marilda, Rosado de Sá. Direito do Petróleo. 3. ed. rev. atual. e ampl. Rio de Janeiro: Renovar, 2018. 
19. Zona Contígua é Faixa entre o mar territorial e o alto-mar, fixada entre 12 e 24 milhas, na qual o Estado exerce sua jurisdição sobre atividades marítimas e sobre diversos interesses nacionais.

20. Plataforma continental é definida como uma faixa de terra submersa, em toda a extensão do litoral do continente. Geralmente, a plataforma possui uma extensão de 70 a 90 km, e profundidade de 200 metros, até atingir as bacias oceânicas.

21. Nota explicativa: Águas adjacentes são aquelas que banham as margens do território de uma nação.

22. ZANELLA, T. V. Curso de Direito do Mar. Curitiba: Juruá, 2013.

23. ZANELLA, T. V. Curso de Direito do Mar. Curitiba: Juruá, 2013.

24. RIBEIRO, Marilda, Rosado de Sá. Direito do Petróleo. 3. ed. rev. atual. e ampl. Rio de Janeiro: Renovar, 2018.

25. REZEK, José Francisco. Direito Internacional Público: curso elementar. 10. ed. rev. e atual. São Paulo: Saraiva, 2005. p.307

26. BRASIL. Decreto no 99.165, de 12 de março de 1990. Convenção das Nações Unidas sobre o Direito do Mar. Brasília: Planalto, 1990. Disponível em: https://www2.camara.leg.br/legin/fed/decret/1990/decreto-99165-12-marco-1990328535-publicacaooriginal-1-pe.html. Acesso em 15 abr.19.

27. GIBERTONI, Carla Adriana Comitre. Teoria e prática do direito marítimo. Rio de Janeiro: Renovar, 1998. p.33.

28. GOMES, Danaciano. Timor Leste: A economia do mar: um contributo para desenvolvimento sustentável. Aveiro: Mare Liberum, 2016. p.51

29. PEREIRA, Eliana Sofia da Silva. Contributo crítico para a compreensão do regime do Mar de Timor à luz do Direito Internacional. 2013. 87 f. Dissertação. (Mestrado em Ciências Jurídicas Internacionais) Faculdade de direito- Universidade 
Nova

de

Lisboa,

2013.

p.56.

Disponível

em:

https://run.unl.pt/bitstream/10362/17481/1/Pereira_2013.pdf. Acesso em: 01 abr. 2019.

30. GOMES, Danaciano. Timor Leste: A economia do mar: um contributo para desenvolvimento sustentável. Aveiro: Mare Liberum, 2016. p.110 e 111

31. RIBEIRO, Marilda, Rosado de Sá. Direito do Petróleo. 3. ed. rev. atual. e ampl. Rio de Janeiro: Renovar, 2018. P. 74 à 80

32. GUSMÃO, Kay Raia Xanana. Breve história do mar do Timor. In: GOVERNO DO ESTADO DO TIMOR LESTE. Novas Fronteiras: conciliação histórica das fronteiras marítimas no mar do timor. Dili: Gabinete das Fronteiras marítimas, 2018.

33. DEL'OMO, Florisbal de Souza. Curso de Direito Internacional Público. Rio de Janeiro: Forense, 2006. p. 292.

\section{TIMOR SEA JUSTICE. All of the contested oil \& gas fields are on EastTimor's} side of the median line ie closer to \#Timor than Australia. Dili, 01 mar. 2016. Tiwitte: @timor sea justice. Disponível em: https://twitter.com/TimorSeaJustice/status/704895304701763584. Acesso em: 25 maio. 2019.

35. GUSMÃO, Kay Raia Xanana. Breve história do mar do Timor. In: GOVERNO DO ESTADO DO TIMOR LESTE. Novas Fronteiras: conciliação histórica das fronteiras marítimas no mar do timor. Dili: Gabinete das Fronteiras marítimas, 2018. p. 34 e 35

36. DEL'OMO, Florisbal de Souza. Curso de Direito Internacional Público. Rio de Janeiro: Forense, 2006. p. 292

37. DEL'OMO, Florisbal de Souza. Curso de Direito Internacional Público. Rio de Janeiro: Forense, 2006. p. 292 
38. REPUBLICA DEMOCRÁTICA DO TIMOR LESTE. História do Timor Leste.

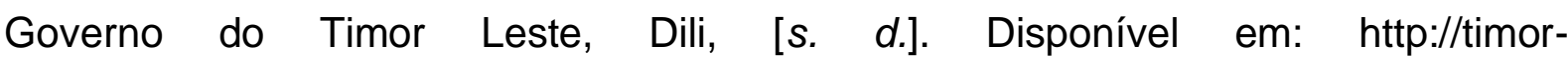
leste.gov.t/ ?p=29\&lang=pt. acesso em: 05 maio 2019.

39. TIMOR LESTE; AUSTRÁLIA. Tratado sobre Fronteiras Marítimas entre Timor Leste e Austrália que estabelece as respectivas fronteiras do mar do Timor Leste. Nova York: [s.n.], 2018. p. 1. Disponível em: http://www.gfm.tl/wpcontent/uploads/2018/03/Port-Timos-Sea-Maritime-BoundaryTreaty_Portuguese.pdf. Acesso em:05 maio 2018.

40. PEREIRA, Eliana Sofia da Silva. Contributo crítico para a compreensão do regime do Mar de Timor à luz do Direito Internacional. 2013. 87 f. Dissertação. (Mestrado em Ciências Jurídicas Internacionais) Faculdade de direito- Universidade Nova de Lisboa, 2013. p.14-15. Disponível em: https://run.unl.pt/bitstream/10362/17481/1/Pereira_2013.pdf. Acesso em: 01 abr. 2019

41. TIMOR LESTE; AUSTRÁLIA. Tratado sobre Fronteiras Marítimas entre Timor Leste e Austrália que estabelece as respectivas fronteiras do mar do Timor Leste. Nova York: [s.n.], 2018. p. 1. Disponível em: http://www.gfm.tl/wpcontent/uploads/2018/03/Port-Timos-Sea-Maritime-Boundary-

Treaty_Portuguese.pdf. Acesso em:05 maio 2018.

42. TIMOR LESTE; AUSTRÁLIA. Tratado sobre Fronteiras Marítimas entre Timor Leste e Austrália que estabelece as respectivas fronteiras do mar do Timor Leste. Nova York: [s.n.], 2018. p. 1. Disponível em: http://www.gfm.t//wpcontent/uploads/2018/03/Port-Timos-Sea-Maritime-Boundary-

Treaty_Portuguese.pdf. Acesso em:05 maio 2018.

43. GIBERTONI, Carla Adriana Comitre. Teoria e prática do direito marítimo. Rio de Janeiro: Renovar, 1998. p. 33.

44. TIMOR LESTE; AUSTRÁLIA. Tratado sobre Fronteiras Marítimas entre Timor Leste e Austrália que estabelece as respectivas fronteiras do mar do Timor Leste. Nova York: [s.n.], 2018. p. 1. Disponível em: http://www.gfm.tl/wp- 
content/uploads/2018/03/Port-Timos-Sea-Maritime-Boundary-

Treaty_Portuguese.pdf. Acesso em:05 maio 2018.

45. TIMOR LESTE; AUSTRÁLIA. Tratado sobre Fronteiras Marítimas entre Timor Leste e Austrália que estabelece as respectivas fronteiras do mar do Timor Leste. Nova York: [s.n.], 2018. p. 1. Disponível em: http://www.gfm.tl/wpcontent/uploads/2018/03/Port-Timos-Sea-Maritime-Boundary-

Treaty_Portuguese.pdf. Acesso em:05 maio 2018.

46. TIMOR LESTE; AUSTRÁLIA. Tratado sobre Fronteiras Marítimas entre Timor Leste e Austrália que estabelece as respectivas fronteiras do mar do Timor Leste. Nova York: [s.n.], 2018. p. 1. Disponível em: http://www.gfm.tl/wpcontent/uploads/2018/03/Port-Timos-Sea-Maritime-Boundary-

Treaty_Portuguese.pdf. Acesso em:05 maio 2018.

47. TIMOR LESTE; AUSTRÁLIA. Tratado sobre Fronteiras Marítimas entre Timor Leste e Austrália que estabelece as respectivas fronteiras do mar do Timor Leste. Nova York: [s.n.], 2018. p. 1. Disponível em: http://www.gfm.tl/wpcontent/uploads/2018/03/Port-Timos-Sea-Maritime-Boundary-

Treaty_Portuguese.pdf. Acesso em:05 maio 2018.

48. GOMES, Danaciano. Timor Leste: A economia do mar: um contributo para desenvolvimento sustentável. Aveiro: Mare Liberum, 2016. p. 59.

49. REPUBLICA DEMOCRÁTICA DO TIMOR LESTE. História do Timor Leste.

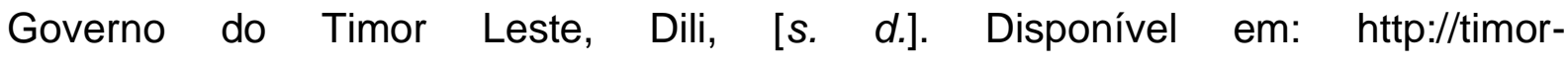
leste.gov.tl/?p=29\&lang=pt. Acesso em: 05 maio 2019.

50. O PRIMEIRO dia no Timor Leste. In: PARCEIROS pela paz. Dili, 06 jul. 2011. Disponível em: https://parceirospelapaz.wordpress.com/category/timor-leste/. Acesso em: 25 maio 2019.

51. REPÚBLICA DEMOCRÁTICA DE TIMOR-LESTE. Ministério das finanças. Orçamento geral do Estado 2018. Díli: Gabinete Ministerial, 2018. p. 7. Disponível 
em: https://www.mof.gov.tl/wp-content/uploads/2018/08/BB5_Port.pdf. Acesso em: 15 maio 2019.

52. TIMOR LESTE; AUSTRÁLIA. Tratado sobre Fronteiras Marítimas entre Timor Leste e Austrália que estabelece as respectivas fronteiras do mar do Timor Leste. Nova York: [s.n.], 2018. p. 1. Disponível em: http://www.gfm.tl/wpcontent/uploads/2018/03/Port-Timos-Sea-Maritime-Boundary-

Treaty_Portuguese.pdf. Acesso em: 19 maio 2018.

53. GOMES, Danaciano. Timor Leste: A economia do mar: um contributo para desenvolvimento sustentável. Aveiro: Mare Liberum, 2016.

54. REPUBLICA DEMOCRÁTICA DO TIMOR LESTE. História do Timor Leste.

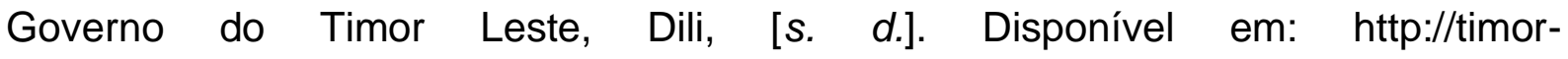
leste.gov.t//p=29\&lang=pt. acesso em: 05 maio 2019

55. TIMOR LESTE; AUSTRÁLIA. Tratado sobre Fronteiras Marítimas entre Timor Leste e Austrália que estabelece as respectivas fronteiras do mar do Timor Leste. Nova York: [s.n.], 2018. p. 1. Disponível em: http://www.gfm.tl/wpcontent/uploads/2018/03/Port-Timos-Sea-Maritime-Boundary-

Treaty_Portuguese.pdf. Acesso em: 19 maio 2018.Acessado: 05/05/19

56. TIMOR LESTE; AUSTRÁLIA. Tratado sobre Fronteiras Marítimas entre Timor Leste e Austrália que estabelece as respectivas fronteiras do mar do Timor Leste. Nova York: [s.n.], 2018. p. 1. Disponível em: http://www.gfm.tl/wpcontent/uploads/2018/03/Port-Timos-Sea-Maritime-Boundary-

Treaty_Portuguese.pdf. Acesso em:05 maio 2018.

57. TIMOR LESTE; AUSTRÁLIA. Tratado sobre Fronteiras Marítimas entre Timor Leste e Austrália que estabelece as respectivas fronteiras do mar do Timor Leste. Nova York: [s.n.], 2018. p. 1. Disponível em: http://www.gfm.tl/wpcontent/uploads/2018/03/Port-Timos-Sea-Maritime-Boundary-

Treaty_Portuguese.pdf. Acesso em:05 maio 2018. 
58. GOMES, Danaciano. Timor Leste: A economia do mar: um contributo para desenvolvimento sustentável. Aveiro: Mare Liberum, 2016

59. GOMES, Danaciano. Timor Leste: A economia do mar: um contributo para desenvolvimento sustentável. Aveiro: Mare Liberum, 2016. p.114-115.

60. GOMES, Danaciano. Timor Leste: A economia do mar: um contributo para desenvolvimento sustentável. Aveiro: Mare Liberum, 2016.

61. REPÚBLICA DEMOCRÁTICA DE TIMOR-LESTE. Ministério das finanças. Orçamento geral do Estado 2018. Díli: Gabinete Ministerial, 2018. Disponível em: https://www.mof.gov.tl/wp-content/uploads/2018/08/BB5_Port.pdf. Acesso em: 15 maio 2019.

62. GOMES, Danaciano. Timor Leste: A economia do mar: um contributo para desenvolvimento sustentável. Aveiro: Mare Liberum, 2016.

Inviato: agosto 2019.

Approvato: dicembre 2019. 